\title{
Editorial for EAIT Issue 4, 2018
}

\author{
Arthur Tatnall ${ }^{1}$
}

Published online: 7 June 2018

(C) Springer Science+Business Media, LLC, part of Springer Nature 2018

In this July issue of the journal of Education and Information Technologies (EAIT) we have articles from researchers in Iran, South Korea, Australia, Croatia, USA, Denmark, Finland, Turkey, South Africa, India and Greece.

The first article: "An empirical study of using sequential behavior pattern mining approach to predict learning styles" comes from Somayeh Fatahi (Iran), Faezeh Shabanali-Fami (Iran) and Hadi Moradi (Iran and South Korea) (https://doi. org/10.1007/s10639-017-9667-1). They points out that learning style of learners are important parameters in his learning process, so that learning styles should be considered in the design, development, and implementation of e-learning environments to increase learners' performance. They propose a sequential pattern mining approach to extract frequent sequential behaviour patterns, which can separate learners with different learning styles.

Following is an article by Therese Keane and William F. Keane from Australia that investigates: "Parents' expectations, perceptions and concerns when schools implement a 1:1 program" (https://doi.org/10.1007/s10639-017-9671-5). The article begins by noting that implementing successful one-to-one computer programs where all students have access to mobile devices throughout the school day requires major stakeholders such as students, teachers and parents to support the initiative, but there is limited literature that explores their expectations, perceptions and concerns. The article reports on a longitudinal study, where parents were surveyed across four years using a mixed methodology. This found a lack of understanding by parents of how their child would learn with the device, that they believed that the devices did not assist the child to be motivated, and that they were a distraction both at school and at home.

"The effect of culture and belief systems on students' academic buoyancy" is by Junu Dahal, P. W. C. Prasad, Angelika Maag and Abeer Alsadoon from Australia and Lau Siong Hoe from Malaysia and notes that learner motivation is implicated in student failure at universities, and this has led to research into internal or external variables that

Arthur Tatnall

Arthur.Tatnall@vu.edu.au

1 Victoria University, PO Box 14428, Melbourne 8001, Australia 
might reduce this failure (https://doi.org/10.1007/s10639-017-9672-4). They found that in the event of academic failure, students' academic buoyancy remains high if they have support through strong cultural connections and from their belief systems. This research contributes to the understanding of the sources of strengths available to international students from primarily collective cultures studying overseas.

Next, Monika Mladenović, Ivica Boljat and Žana Žanko from Croatia present: "Comparing loops misconceptions in block-based and text-based programming languages at the K-12 level" (https://doi.org/10.1007/s10639-017-9673-3). Novice programmers face difficulties while learning to program but most studies are conducted at the undergraduate level and few at elementary schools (K-12). The authors question whether misconceptions about loops, in programming, at elementary school level are similar to those at the undergraduate level. Their research asked if we prevent the misconceptions by using a different pedagogical approach with visual programming languages and shifting the programming context toward game programming.

“Enhancing future K-8 teachers' computational thinking skills through modelling and simulations" by Rachel F. Adler and Hanna Kim from the USA points out that it is now important for teachers to incorporate computational thinking (CT) into their science classes (https://doi.org/10.1007/s10639-017-9675-1). Their article describes how they modified existing structures of a science methods course for preservice teachers to include CT via modelling and simulations. They describe a programmed an animated model of the solar system (using Scratch), and a web-based simulation to visualize Newton's second law of motion with a dynamic graph feature.

The article that comes next: "A review of the use of virtual reality head-mounted displays in education and training", was written by Lasse Jensen and Flemming Konradsen from Denmark. In the light of substantial recent improvements to the quality and availability of virtual reality (VR) hardware their work involved searching for documents reporting on experimental studies to update knowledge about the use of head-mounted displays (HMDs) in education and training (https://doi.org/10.1007 /s10639-017-9676-0). Their review identified a number of situations where HMDs are useful for skills acquisition, including cognitive skills related to remembering and understanding spatial and visual information and knowledge, psychomotor skills related to head-movement such as visual scanning or observational skills and affective skills related to controlling an emotional response to stressful or difficult situations.

"Lines, roamers, and squares: Oh my! using floor robots to enhance Hispanic students' understanding of programming" by J. Elizabeth Casey, Puneet Gill, Lisa Pennington and Selina V. Mireles from the USA, looks at teaching programming and coding skills in K-12 classrooms and how this is becoming a part of science, technology, engineering, and maths (STEM) programs across the United States (https://doi. org/10.1007/s10639-017-9677-z). They point out that these are often available through extra-curricular activities such as Robotics club, maths club, STEM club, etc. and that increasing STEM opportunities for students who are English language learners, culturally and linguistically diverse learners, and students from underserved backgrounds is vital. The article describes activities they developed.

Next, "Assessing alignment between information technology educational opportunities, professional requirements, and industry demands" points out that information technology and computing are growing fields, offering far more job 
opportunities than applicants, but that little data is available to indicate how course content, employer needs and additional learning opportunities can work together the prepare graduates to enter the IT workforce (https://doi.org/10.1007/s10639017-9678-y). The authors are Marcia A. Mardis, Jinxuan Ma, Faye R. Jones, Chandrahasa R. Ambavarapu, Heather M. Kelleher, Laura I. Spears and Charles R. McClure from the USA. Their article used text analysis to examine the extent to which course syllabi, job postings, internship postings and industry certifications in undergraduate information technology preparation programs commonly reflected national IT curriculum knowledge areas.

"Birth, life and death of the Victorian Education Ultranet" describes a major ICT infrastructure project in the education system of the Australian State of Victoria (https://doi.org/10.1007/s10639-017-9679-x). Contributed by Arthur Tatnall and Bill Davey from Australia it examines the development and ultimate demise of a very large $(\$ 180 \mathrm{~m})$ project, intended for 1500 schools and called the Ultranet. The Ultranet was a Web-based online system designed to support the delivery of curriculum, online teaching and learning and the sharing of knowledge across all Victorian Government schools. The study traces the design and implementation of the project as a sociotechnical innovation in education. The study found that potential benefits from a very large project like this can be nullified by a number of factors including: change in sponsorship of the project, an over emphasis on security concerns, failure to enrol crucial stakeholders and a naive assumption by technologists that technology with potential benefits will always be accepted. The Victorian Government abandoned the Ultranet in June 2013.

Nicholas Mavengere and Mikko Ruohonen from Finland follow with: "Context and user needs in virtual learning in pursuit of qualities of learning" (https://doi. org/10.1007/s10639-017-9681-3). This research sought to promote virtual learning experience and investigated virtual learning at a master's level information and communication technology for development (ICT4D) course in 2016. The course was offered twice and to two sets of different student groups and aimed to investigate how well the virtual environment and pedagogy promoted qualities of learning including active, constructive, collaborative, intentional, contextual, transfer and reflective learning. The results of their study include highlighting of pedagogical techniques and technological tools that fit the learners' needs and study content requirements to foster learning in a virtual environment.

Coming up next is an article by Fatma Burcu Topu, İlknur Reisoğlu, Turkan Karakus Y1lmaz and Yuksel Göktaş from Turkey (https://doi.org/10.1007/s10639-017-9683-1). Titled: "Information retention's relationships with flow, presence and engagement in guided 3D virtual environments", it describes a study aimed at examining the correlation of different variables with information retention in guided 3D virtual learning environments by the employment of three experimental designs. Information retention test, flow, presence and engagement scales were used as data collection tools. They found that focused attention, enjoyment and skill dimensions have a weak but significant correlation with information retention rather than the total score of flow, the challenge has a moderate and significant negative correlation.

The next article: "Affordances of mobile devices and note-taking apps to support cognitively demanding note-taking" by Mari van Wyk and Linda van Ryneveld from South Africa, argue that as note-taking is one of the more common and ever-present 
learning activities that form an important part of all students' daily lives, the potential of using technology to enhance note-taking activities has recently come under the spotlight (https://doi.org/10.1007/s10639-017-9684-0). They argue that while mobile technologies are useful for the value they can add to students' learning experiences, they can easily become a distracting factor, rather than the improvement they were intended to be. Their article aims to provide insight into the affordances of mobile devices and note-taking applications, in order to support cognitively demanding notetaking.

Automatically assessed exercises with immediate feedback can be a powerful tool for enhancing the effectiveness of education according to Mikko-Jussi Laakso, Erkki Kaila and Teemu Rajala from Finland. In their article: "ViLLE - collaborative education tool: Designing and utilizing an exercise-based learning environment" they discuss the design and implementation of a collaborative learning tool called ViLLE (https://doi.org/10.1007/s10639-017-9659-1). ViLLE includes various different exercise types which were designed to assist in the learning of computer science, mathematics and other subjects. It also supports different learning and teaching methods, such as pair programming and peer review.

"Data mining based analysis to explore the effect of teaching on student performance" comes from Anupam Khan and Soumya K. Ghosh from India (https://doi.org/10.1007/s10639-017-9685-z). Prediction of student performance may be helpful for both teacher and the student, but influencing factors of the student performance need to be identified first to build up such early prediction model. They outline how superior teaching acts as a catalyst which improves the knowledge dissemination process from teacher to the student and also motivates the student to put more effort into study. In this article they propose a quantifiable measure of improvement with respect to the expected performance of a student. Their data mining technique explores academic records collected from an online system of an academic institute of national importance in India.

"Transformative learning in designing algorithms for reporting information systems" describes a teaching method that enables students to design efficient and effective algorithms for reporting information systems applications. By Anand Jeyaraj (USA), it describes how adopting the general principles of transformative learning, students are immersed in a series of activities and interventions to enable learning (https://doi.org/10.1007/s10639-018-9687-5). The interventions allowed students to gain knowledge about the output data structures such as list, table and cube, the sequence control structures that may take different execution times and the required assumptions such as source data tables and mapping rules which can contribute to the design of efficient and effective algorithms.

Fatma Betül Kurnaz, Esin Ergün, and Hale Ilgaz from Turkey then write: "Participation in online discussion environments: Is it really effective? (https://doi. org/10.1007/s10639-018-9688-4)" Their study aimed to develop a rubric to assess participation of students in online discussion environments. The rubric consists of two parts: 'Form and Content', and 'Number and Density', and seven criteria in total. 'Form and Content' consists of congruity of the message in terms of subject, clarity of the message, original value of the message, interactional value of the message, and directing the subject. 'Number and Density' consists of the number of messages and their density. Their findings showed that the graded scoring key is reliable and valid. 
The last article in this issue: "Learning activities as enactments of learning affordances in MUVEs: A review-based classification" comes from Olga Mantziou, Nikiforos M. Papachristos and Tassos A. Mikropoulos from Greece (https://doi. org/10.1007/s10639-018-9690-x). Three Dimensional Multi-User Virtual Environments (MUVEs) are promising tools in education because of the unique affordances they offer but these learning affordances imply certain actions that in turn can lead to corresponding learning activities. The authors indicate that there seems to be a lack of reports on which of the affordances of MUVEs for learning and teaching are used and how they are enacted by relevantly designed learning activities. Their reported study investigated the learning activities conducted in Second Life, the most popular and widely used among the 'sandbox' type MUVE platforms, as reported in 205 empirical studies, by associating them with the learning affordances they enact.

As Editor-in-Chief of this journal I am keen to see articles on all aspects of the use of Information Technologies in Education from the micro to the macro and from theory to practice. I hope to see articles from Education Faculty academics, School Teachers, Educational Administrators, academics from Computer Science and Information Systems and from others interested in any aspect of the use of computers in education. It would also be good to see articles covering an even greater diversity of topics relevant to Education and Information Technologies and from an even wider range of contributors.

\section{Arthur Tatnall}

Editor-in-Chief 